\title{
Être plurilingue : combinaisons et complémentarité
}

Federica Diémoz

\section{OpenEdition}

\section{Journals}

Édition électronique

URL : https://journals.openedition.org/esp/4773

DOI : $10.4000 /$ esp.4773

ISSN : 2532-0319

\section{Éditeur}

Centre d'Information sur l'Éducation Bilingue et Plurilingue

\section{Édition imprimée}

Date de publication : 1 décembre 2019

Pagination : 91-96

ISSN : 1127-266X

\section{Référence électronique}

Federica Diémoz, «Être plurilingue : combinaisons et complémentarité », Éducation et sociétés

plurilingues [En ligne], 47 | 2019, mis en ligne le 01 septembre 2021, consulté le 01 septembre 2021.

URL : http://journals.openedition.org/esp/4773 ; DOI : https://doi.org/10.4000/esp.4773 


\section{ÊTRE PLURILINGUE: COMBINAISONS ET GOMPLÉMENTARITÉ (1)}

\section{Federica DIÉMoz}

Federica Diémoz (borm 1975) comes from Roisan, a village near Aosta, town in the Aosta Valley, an autonomous and bilingual region in the North-West of Italy. Her mother tongue is the Francoprovençal patois, a Gallo-Roman language. Entering school meant for her learning good Italian and correct French, the first being dominant. The patois, looked down upon and banned from the schools, remained the language of the family and the neighborhood. The gap between her public school life and her private life grew larger. At the University, she went on to study French, English, Latin and especially, she discovered dialectology, a subjet matter that allowed her to get back in touch with her origins. After a dissertation on the Roisan Patois, she left for Neuchâtel (Switzerland); to start a Ph.D. on the Francoprovençal tongues spoken in the Aosta Valley. The patois, which before she could only whisper in, is now the respecled and valued object of her research.

Federica Diémoz (1975) è originaria di Roisan, un piccolo comune della Valle d'Aosta, regione autonoma bilingue del nord-ovest dell'Italia. La sua lingua materna è un dialetto francoprovenzale, lingua gallo-romanza. Sin dai primi anni di scuola impara l'italiano e il francese con una netta predominanza del primo. Il patois, connotato negativamente nelle scuole, rimane comunque la lingua della famiglia. Si crea cosi un abisso tra la vita scolastica e la realtà quotidiana. All'Università studia il francese, l'inglese, il latino, ma soprattutto scopre la dialettologia, disciplina che le permette di riallacciare $i$ contatti con le sue origini. Dopo una tesi di laurea sul "patois" di Roisan, parte per Neuchâtel (CH) dove sta preparando una tesi di dottorato sui dialetti francoprovenzali della Valle d'Aosta. Il "patois", un tem po sussurato, è adesso rispettato e valorizzato nelle sue ricerche.

Parole-chiave: autobiografia, testimonanzia, francoprovenzale lingua materna, dialettologia, tesi sul "patois"

Key-zords: autobiography, testimony, francoprovençal mother tongue, dialectology, thesis on "patois"

"Mé prédjo patoué é si de Rèisàn": voilà une phrase qui résume mes (premières) années d'enfance. "Moi je parle le patois et je suis de Roisan". Cet énoncé véhicule plusieurs informations. Tout d'abord, le patois de Roisan est ma langue maternelle. En Vallée d'Aoste, on utilise le terme "patois" pour désigner la langue indigène parlée dans un endroit précis, sans associer à ce mot des connotations péjoratives. Ma langue maternelle fait partie des parlers francoprovençaux. Le domaine francoprovençal recouvre la Vallée d'Aoste, quelques vallées piémontaises, la Suisse romande, à l'exception du nord du Jura, et une région 


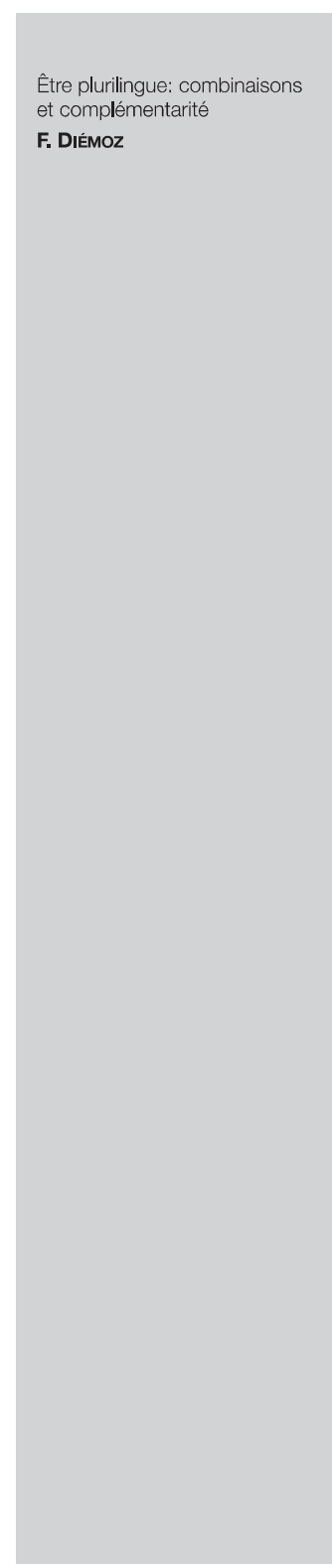

française englobant la Savoie, le sud du Jura français, le nord du Dauphiné et l'est de la Bresse. Ce territoire se trouve ainsi à la charnière des domaines d'oil et d'oc et aux confins des parlers italo-romans.

L'aire francoprovençale n'a jamais connu d'unité politique et identitaire, le francoprovençal ne s'est pas développé en tant que langue littéraire. Cette langue est ainsi caractérisée par une forte fragmentation interne et par une absence de normalisation. Par exemple, dans ma région, chaque village a sa variété de parler, variété qui se différencie par des particularités phonétiques et lexicales. Je me souviens que j'avais trois-quatre ans quand j'ai entendu mes cousines répondre à leur maman "oi". J'avais bien compris qu'elles voulaient dire "oui" et que leur "oi" correspondait à mon "oué", mais ce fait m'avait frappée. Cela explique aussi pourquoi à propos des patois on explicite exactement le lieu de provenance, et l'énoncé initial en est un exemple.

Roisan est un village de 700 habitants, situé à sept kilomètres de la ville d'Aoste dans la vallée du Grand Saint-Bernard en montant vers le col pour aller en Suisse. C'est dans cet endroit que j'ai grandi et que j'ai suivi l'école maternelle et l'école élémentaire. A cette époque, vers la fin des années 70, le parler francoprovençal était la seule langue de la famille et du voisinage. L'italien commençait à apparaître dans ma vie par le biais des médias, mais, vivant à la campagne, je préférais jouer dehors plutôt que regarder la télévision. Ainsi, j'ai commencé l'école maternelle à trois ans en n'ayant que le patois comme langue active. L'entrée à l'école a signifié l'apprentissage de l'italien et du français. Etant donné que la Vallée d'Aoste est une région bilingue, on apprend ces deux langues depuis les premières années scolaires. Cependant, le patois n'est pas reconnu comme langue officielle, il n'est pas non plus admis comme langue véhiculaire dans les écoles d'où souvent il est banni. Je me souviens, tout de même, que l'institutrice, dans certains moments difficiles (un enfant en colère ou en larmes), s'adressait à nous en patois. Ce choix linguistique créait une ambiance de confiance et de complicité qui dépassait le cadre scolaire. Il faut aussi dire que, à Roisan, vers les années 1980, 80 \% des enfants étaient patoisants. Si les langues d'enseignement étaient l'italien et le français, avec une petite prédominance de la première, la langue véhiculaire entre les enfants restait le patois.

Puis à l'école élémentaire, l'italien s'est renforcé comme langue d'enseignement et aussi comme langue véhiculaire. Entre copains, on continuait à parler patois, même si l'arrivée d'enfants 


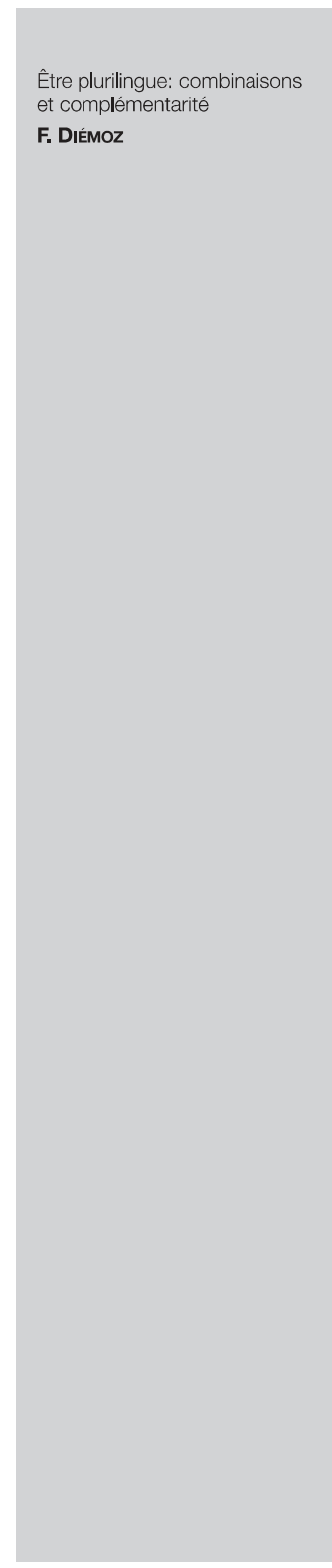

italophones nous poussait à introduire de plus en plus l'italien. Les institutrices, même si elles étaient originaires de la région, ne parlaient jamais patois avec nous mais, au moins, elles ne nous l'interdisaient pas. Elles craignaient que ce code interfère dans l'apprentissage du bon italien et du français correct. Je me rappelle que certains copains patoisants avaient de la peine à apprendre l'italien et que, spontanément, ils faisaient des calques sur le patois. Ce phénomène était très mal perçu et on leur disait: "c'est du patois, c'est faux!" Au lieu d'expliquer que cette adaptation patoise n'avait aucun sens dans un contexte italien, on culpabilisait l'enfant, qui finissait par associer au mot patois la notion de fausseté. Quel plaisir j'ai eu quand, il y a deux ans, j'ai lu dans une réserve d'indiens en Arizona, l'affiche suivante:

"What we do in school must not insult the children's past but must build upon their past and encourage future learning" (Sigmund A. Boloz, 1999).

Si à l'école maternelle (de trois à cinq ans) les activités se déroulaient dans les deux langues, à l'école élémentaire (de six à dix ans), j'ai appris le français comme L2; les systèmes d'enseignement bilingue n'étaient pas encore au point à ce moment-là. On ne parlait français que pendant les heures d'enseignement de cette langue et, en dehors de l'école, je n'avais pas beaucoup d'occasions pour le pratiquer. Toutefois, pendant l'été, je pouvais exercer mon français avec des enfants parisiens, fils d'émigrés valdôtains, qui passaient leurs vacances à Roisan. Je me souviens que, les premiers jours, j'avais de la peine à trouver les mots qu'on utilise dans la vie de tous les jours ou à comprendre des jeux de mots ou des expressions qu'on n'apprend pas sur les bancs de l'école. Quels beaux moments où les mélodies françaises, patoises et italiennes alternaient harmonieusement! Le passage d'un code linguistique à l'autre était fait de façon naturelle. Etant donné mon envie d'apprendre les langues, souvent je devais traduire du français en patois ou passer du français à l'italien pour des enfants italophones en vacances dans mon village. Cette activité, perçue comme amusante, s'est très tôt révélée difficile et insatisfaisante. Souvent je disais: "je ne peux pas dire cette chose de la même manière ou c'est pas tout à fait la même chose". Ces quelques remarques, spontanées à l'époque, ont été par la suite confirmées par les cours de traduction suivis à l'université.

Pendant les années de l'école moyenne (de onze à treize ans), le rôle de la langue italienne a continué à se renforcer dans la vie scolaire et quotidienne. Le patois restait la langue de la famille et de la vie communautaire dans les villages valdôtains. A l'école, à 


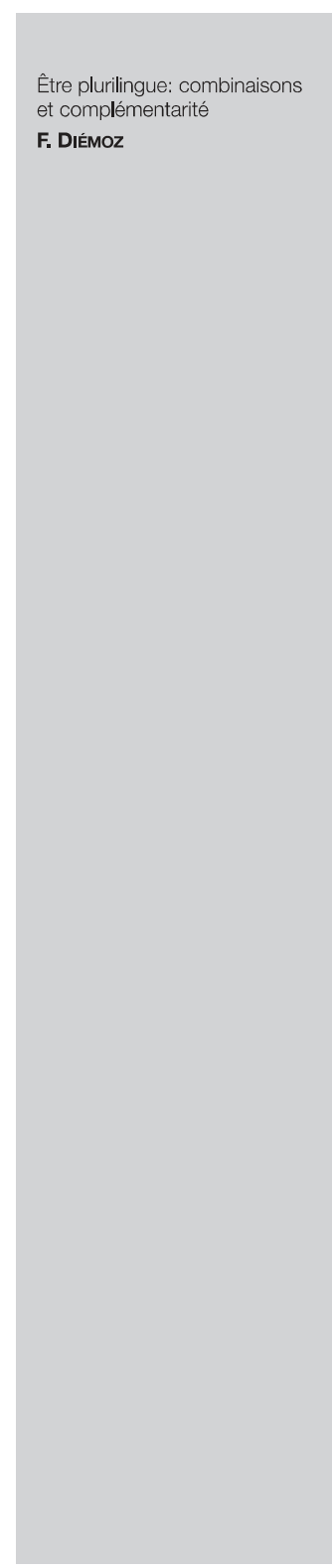

l'exception des copains d'enfance, c'était l'italien qui était choisi. Le français était perçu comme langue littéraire et il fallait surtout bien apprendre la grammaire et l'orthographe.

L'école normale à Aoste (de quatorze à dix-huit ans) a marqué une rupture avec mes origines et ma langue maternelle. Non seulement le patois était moins pratiqué en ville que dans les villages, mais il était dévalorisé et le fait de parler cette langue était associé à la grossièreté, à la vie paysanne, à une culture inférieure. A l'école, même avec des nouveaux copains patoisants, on parlait italien. Ma soeur aussi, quand elle était à Aoste, était gênée de parler patois. Je lui ai toujours répondu en patois, mais je dois avouer que souvent c'ćtait en chuchotant.

L'université devait creuser encore plus le fossé entre ma vie scolaire et ma vie privée. Mais, à ce moment-là, l'envie d'aller à l'université pour apprendre de nouvelles matières, en particulier des langues, était telle que même le départ de Roisan et l'éloignement de la famille n'étaient pas dramatiques. J'ai passé quatre ans à Turin, de dix-neuf à vingt-trois ans, où j'ai suivi des cours de langues et littératures étrangères. Je voulais perfectionner mes connaissances dans la langue française et développer mon anglais, appris superficiellement jusqu'à ce moment-là. Par la suite, j'ai découvert une passion pour la linguistique et la philologie romane et j'ai préféré ces disciplines à la littérature. J'étais bien contente d'avoir fait du latin et j'appréciais cette langue apprise si rigoureusement à l'école secondaire.

C'est en suivant ces intérêts que j'ai découvert la dialectologie, un tournant dans ma vie. Pendant ces années turinoises, j’utilisais majoritairement l'italien, le français et l'anglais pendant les cours et le patois au téléphone avec ma famille ou quand je restais en Vallée d'Aoste. Dans ma formation traditionnelle-puriste, on apprenait la langue italienne, $l e$ français, $l$ anglais et le concept de variation n'était pas présent. Je ne pouvais pas imaginer qu'à l'université on puisse s'intéresser à des codes linguistiques qui n'appartiennent pas à la norme, dans ce cas les dialectes italiens. Et quelle surprise quand j'ai appris que ma langue maternelle aussi était prise en considération et faisait l'objet de recherches! En étudiant cette matière, en comprenant l'origine des langues, leurs diversités internes, j’ai appris à respecter et à valoriser mon bagage culturel. J'avais l'impression de renouer des contacts et je ne m'étais pas encore rendue compte à quel point cet aspect était important pour moi. J'ai ainsi décidé de faire un mémoire en dialectologie sur un sujet ethnolinguistique. Il s'agissait d'une recherche ethnographique dans mon village d'origine, où j'inter- 


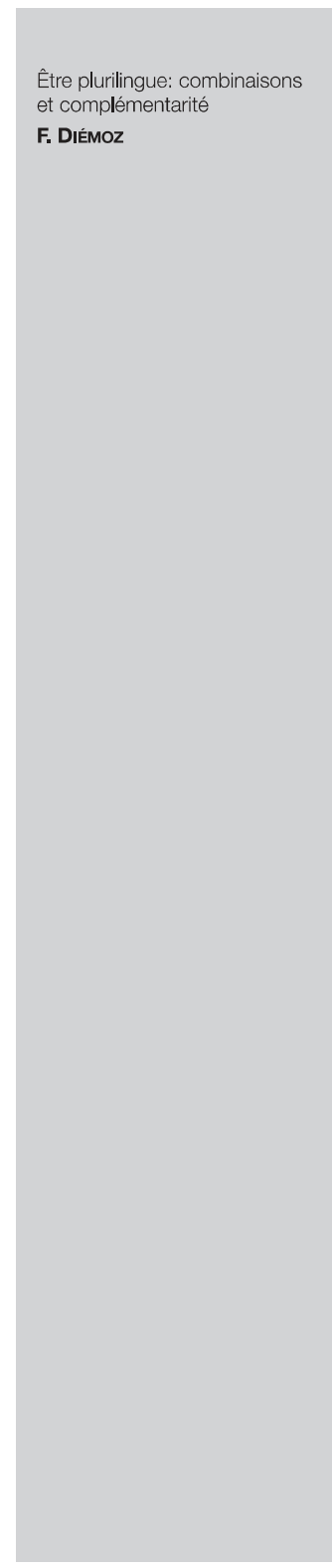

viewais en patois les habitants, transcrivais les entretiens en patois et traduisais ces textes en français. Des remarques sur les particularités de mon parler francoprovençal complétaient le travail. $\mathrm{Ce}$ mémoire m'a donné la possibilité de faire un lien entre la vie réelle et le monde scolaire, deux réalités qui, auparavant, avaient toujours été séparées. Je ne pouvais pas savoir que cette recherche n'était pas un achèvement mais un début. En effet, après avoir terminé mes études universitaires, en 1999 j'ai accepté un travail de recherche au Centre de dialectologie de l'Université de Neuchâtel, en Suisse. J'étais engagée dans un projet transfrontalier Italie-Suisse pour la réalisation d'un atlas linguistique audiovisuel du francoprovençal valaisan. Je travaillais sur les points d'enquête valdôtains insérés dans ce projet. Ma langue maternelle était au centre des intérêts, elle était mise sur le même plan que les autres langues romanes, étant donné son origine également "noble". J'ai toujours continué à parler patois avec ma famille et avec mes amis valdôtains, mais surtout, c'est le voile de gêne et d'infériorité qui a disparu. Actuellement, je prépare un doctorat de recherche sur les parlers francoprovençaux valdôtains.

A Neuchâtel, ville francophone, le concept de variation géographique, interne à chaque code linguistique, s'est consolidé. Plusieurs variétés de français y vivent et font l'objet d'études: le français régional de la Suisse romande, les français régionaux de la France, le québécois, le français en Afrique. J'ai été surprise d'entendre "septante" à la place de "soixante-dix", choix qui aurait fait trembler mes professeurs de français. Mais ici en Suisse on utilise régulièrement "septante", mot qui est perçu comme régional mais qui ne porte pas de connotations.

Dans cette ville, j'entends également parler des variétés de l'italien: le tessinois, l'italien des immigrés.

En outre, le fait de connaitre ces trois langues romanes (le patois, l'italien et le français) et leurs différentes facettes m'a aidé dans la compréhension d'autres langues néo-latines: l'espagnol, le catalan et l'occitan. Par contre, je me suis sentie vraiment dépaysée quand j'ai voulu découvrir une langue celtique, le breton. Quelle difficulté à plonger dans un monde où on n'a pas de repères et de termes de comparaisons!

Mon prochain but est l'apprentissage de l'allemand, que je déchiffre avec peine. Les connaissances d'une autre langue germanique, l'anglais, pourraient faciliter cette conquête.

Depuis quatre ans, j'effectue des allers et retours entre la Vallée d'Aoste et Neuchâtel. Au Centre de dialectologie, je travaille sur les langues gallo-romanes et j’utilise comme langues véhiculaires 


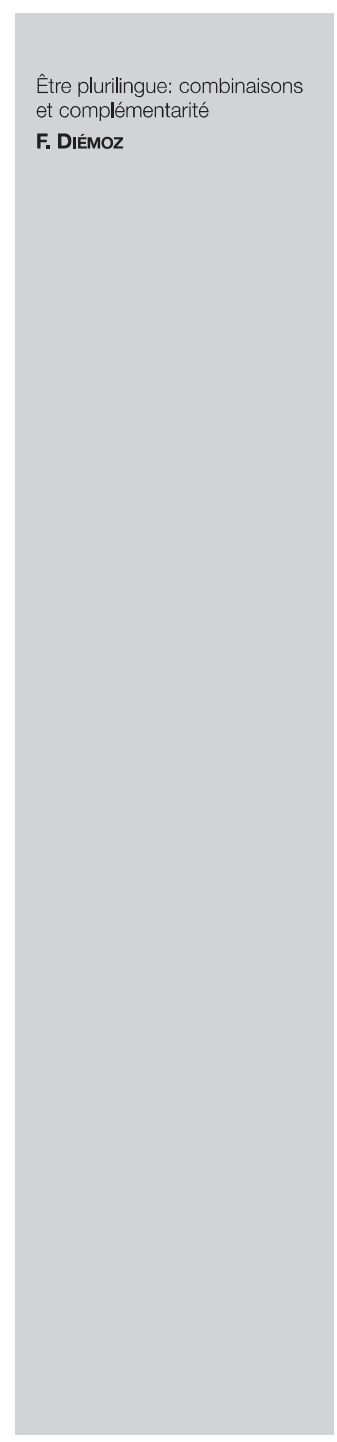

le français et l'italien (une de mes collègues a vécu pendant longtemps en Italie). Je suis ainsi constamment en train de passer d'un code à l'autre et, souvent, je me dis: "en italien j'ai un mot qui explique mieux ce concept" ou "en français il y a cette locution qui rend vraiment l'idée" ou encore "en patois j'ai différents verbes qui indiquent cette action, mots que je ne connais ni en italien ni en français". Souvent dans une conversation en italien, j'aurais envie d'utiliser un mot français parce qu'il exprime vraiment mon idée dans un contexte donné; j'essaie cependant d'employer le correspondant italien, (je ne parlerais pas de traduction), souvent c'est de la paraphrase, mais une nuance manque quandmême. Faut-il considérer ces phénomènes comme des manques, des vides dans mes connaissances linguistiques? Je les considère plutôt comme le résultat de la combinaison de codes et de leur complicité. Sur la base de ces faits, depuis quelque temps, je me sens plurilingue, passant régulièrement du patois à l'italien et au français. Avant mon arrivée à Neuchâtel, si je devais répondre à la question "Etes-vous plurilingue?" je répondais "non, plutôt bilingue italien-patois"; je ne discuterai pas ici des notions de bilinguisme et de diglossie. Maintenant, une variété de français fait partie de ma vie, non seulement j'utilise ce code couramment, mais je pense dans cette langue. Il m'arrive, d'ailleurs, de me trouver dans des magasins en Italie et de réfléchir en français ou de commencer à poser une question en français.

Mais le patois reste la langue intime, des sentiments et des rêves.

Exprimer ses propres ressources ne signifie pas s'enfermer dans un petit monde, mais au contraire, cela permet de mieux apprécier les diversités linguistiques et culturelles. Si les sentiments et les désirs modèlent les contours de notre vie, les langues colorent et complètent ce patchwork, où les nombreuses teintes se combinent... harmonieusement.

NOTE

Article paru dans Éducation et Sociétés Plurilingues n ${ }^{\circ} 14$ en juin 2003. 